\title{
PREFACE AND INTRODUCTION
}

This symposium was held at the Collège de France in Paris from August 31 to September 4, 1970. The Organizing Committee consisted of V. Bumba, R. Howard (Chairman), K. O. Kiepenheuer, R. Michard, E. N. Parker, A. B. Severny, V. E. Stepanov, and T. Takakura. The Local Organizing Committee consisted of Miss G. Drouin (Secretary), R. Michard (Chairman), J. -C. Pecker, and J. Rayrole.

We are indebted to the Collège de France for their kind hospitality. I wish to express my gratitude to members of the Organizing Committee for advice and assistance and to R. Michard and the Local Organizing Committee, who were responsible for the smooth running of the sessions, the distribution and collection of the discussion sheets, and for a delightful Wednesday afternoon excursion to Meudon. It is a pleasure to thank J. W. Evans, V. E. Stepanov, K. O. Kiepenheuer, R. G. Giovanelli, T. G. Cowling, V. Bumba, W. C. Livingston, and J. M. Wilcox who kindly served as session chairmen. I also wish to thank Miss Judy Harstine and John M. Adkins of the Hale Observatories, for invaluable assistance in editing the proceedings. This Symposium has been supported financially by the International Astronomical Union.

An IAU Symposium on Stellar and Solar Magnetic Fields (No. 22) was held in Rottach-Egern near Munich, Germany just seven years ago, in 1963. At that time we could devote a symposium to both stellar and solar magnetic fields and still maintain a rather comfortable and uncrowded program. Now the subject of solar magnetic fields has grown so much that it is too big for one symposium. More than 160 persons attended IAU Symposium No. 43 in Paris, and there were 94 papers of various lengths. A number of papers which authors wished at the last moment to present had to be refused because of lack of time. If work in the field continues at this rate, future symposia will have to cover only part of the subject of solar magnetic fields.

An interesting measure of activity in the field is the number of photoelectric magnetographs which contributed results to the symposium. In the case of the RottachEgern meeting this number was three. In the case of IAU Symposium No. 43, I count at least 15 such instruments.

Growth in the subject of solar magnetic fields has proceeded in several subdisciplines. On the smallest scale a number of investigators have looked at the smallest known magnetic features on the Sun. Results have been somewhat disparate, but all agree that outside sunspots at least a large fraction of the magnetic flux is contained in small $(\sim 1000 \mathrm{~km})$ bundles of lines of force. The magnetic field within such features ('gaps' or 'knots') may be expected to reach values of at least a few hundred gauss. High temperatures within such features tend to weaken many Fraunhofer lines commonly used to measure magnetic fields. The resulting line-profile changes adversely affect the signals one gets from most photoelectric or photographic magnetic 
field measuring techniques. The physics of these 'gaps' and their relation to the shortlived pores and the larger structure of the supergranular network and active regions are areas of intensive investigation at a number of observatories. The recent suggestion that magnetic fields in 'gaps' may be quantized is an intriguing one, and no doubt will be followed up by many investigators.

On a somewhat larger scale the magnetic structure of the supergranular network and active regions continues to be an active field for research. Several investigators in recent years have accurately measured the supergranular velocity fields and have associated magnetic field concentrations with the boundaries of the calcium network. The problem of exactly what association exists, if any, between flares and magnetic field changes in active regions continues after many years essentially unanswered. Many astronomers at several observatories continue to pursue this topic. Part of the problem has been a disappointingly inactive cycle for flares. Some high-resolution magnetic cine films obtained within the last year or so give promise of solving this problem in the near future - although in the next few years these beautiful observations will probably raise more questions than they will solve.

On the large scale, advances have been made in recent years in the association of photospheric magnetic fields extended above the photosphere with coronal features and with the interplanetary magnetic field in the neighborhood of the Earth. The large-scale distribution of magnetic fields on the solar surface has been studied extensively in recent years. The dynamics of the large-scale fields appear to be explainable at least in part by a combination of the eroding effects of supergranular motions on the active region magnetic fields and the shearing effects of differential rotation. The failure of the polar magnetic fields to reverse their polarities so far in this cycle has provided an interesting new factor to consider in connection with theories of the solar cycle.

The subject of solar magnetic fields has developed rapidly in recent years, and we can look forward to exciting advances in the years to come.

Hale Observatories, Carnegie Institution of Washington, ROBERT HOWARD California Institute of Technology, Pasadena, Calif., U.S.A. 